\title{
Fluid Frame Magneto-Hydrodynamic Antenna
}

\author{
Rajveer Singh Yaduvanshi* \\ ECE Department \\ AIT, Delhi, India -110031 \\ Harish Parthasarathy \\ ECE Department \\ NSIT,Govt of Delhi,India
}

\author{
Asok De \\ Principal,AIT \\ Delhi,India
}

\author{
Richa Gupta \\ ECE Deptt,MSIT \\ Delhi,India
}

\begin{abstract}
A rotating fluid frame Antenna consisting of conducting fluid (saline water) is radiating under electric field and magnetic field conditions. Resonant frequency depends on volume of fluid. The prototype model is presented with analytical solution of MHD cylindrical coordinates method. The radiation patterns of the antenna have been function of conducting fluid velocity $\mathrm{v}$, magnetic field intensity $\mathrm{H}$ and electric fields intensity $\mathrm{E}$.
\end{abstract}

Index Terms - Magneto hydrodynamics, Maxwell's Equation, Navier Stokes Equation, Fluid Frame, Adaptable Antenna .

\section{INTRODUCTION}

The word magneto hydrodynamics (MHD) is derived from magneto- meaning magnetic field, and hydro- meaning liquid, and -dynamics meaning movement [1-3]. MHD is the study of flow of electrically conducting liquids in electric and magnetic fields. Ting and King determined in 1970 that dielectric tube can resonate. It has been shown that conducting fluid oscillates under the influence of electromagnetic field conditions. This conducting fluid can operate as antenna at microwave frequencies. Magnetohydrodynamic Antenna, using electrically conducting fluid (saline water $\mathrm{NaCl}$ ) under controlled electromagnetic fields is presented. Rotating fluid frame provides excellent coupling of RF energy into fluid. RF metallic probe makes direct contact with the rotating fluid frame, hence, eliminates any possibility of air gap. Fluid resonator volume, chemical properties of fluid, electric field and magnetic field are the factors of resonant frequency, gain and return loss[4-6]. We shall model an MHD antenna to resonate at some specific resonant frequency. It is can be tuned in wide range i.e. 1- 10 GHz. Fluid permittivity depends on type of conducting fluid and its dielectric constant.

The Maxwell's equations, Navier Stokes equations and equations of mass conservation for the conducting fluid and EM field have been set up. These are expressed as partial differential equations for the Stream Function, Electric and Magnetic fields. These equations are first order in time, which helps us to predict velocity field of the fluid in the near field region and electromagnetic field in the far field region[8-10]. Adding salt to water is believed to break the structure of water clusters, freeing molecules which in turn have a lower relaxation time, thus causing an overall polarizing effect in the bulk solution [11-12]. When the saline water (or electrolyte) is biased with electrodes of opposite polarity, the negative electrode attracts the hydrogen atoms while the positive electrode attracts the oxygen atoms. This accelerates the ion transport mechanisms and results in the electrolysis process at the solution. Water molecules oscillate due to magneto hydrodynamic process and impact ionization takes place inside the fluid. Measurements for Resonant Frequency, Return Loss, Gain and Radiation Pattern have suggested at different fluid parameters i.e. salinity, height and volume of conducting fluid [13-14]. In this work, we propose to design, develop, formulate and fabricate a prototype MHD antenna with adaptability in resonant frequency, permittivity and permeability. It can be best suitable for Military use, as plasma become conductive only in excited state.

\section{FORMULATIONS}

The equation of motion of a fluid in a uniformly rotating frame with angular velocity $\omega$ is given by Navier Stokes Equation. Here $\sigma$ Conductivity, $\rho$ Mass density, $p$ Pressure, $v$ Kinetic viscosity, $v$ Velocity of fluid ,E Electric Field, J Current Density, B Magnetic Field, $\eta$ Fluid Viscosity and $\psi$ Stream Function.

$\mathrm{F}$ Lorentz force $=\mathrm{J} X \mathrm{~B}$ and $\mathrm{J}=\sigma(\mathrm{E}+\mathrm{v} \times \mathrm{B})$, As we know Navier Stokes equation is $\rho(v \cdot \nabla v+v, \mathrm{t})=-\bar{\nabla} p+\eta \nabla^{2} \mathrm{~V}+\mathrm{J} \times \mathrm{B}$

$v, \mathrm{t}+v \cdot \nabla_{y}+2 \omega \times v+\omega \times(\omega \times \mathrm{r})=$ $-\frac{v_{p}}{e}+v \nabla^{2} v$

Assuming the flow to be two dimensional and fluid to be incompressible, we obtain an equation for the stream function. Here $v$ is kinetic viscosity of the fluid.

$v=v_{x}(\mathrm{t}, \mathrm{x}, \mathrm{y}) \hat{x}+v_{x}(\mathrm{t}, \mathrm{x}, \mathrm{y}) \hat{y}$

Angular velocity $\quad \omega=\omega_{0} z$

$v_{x}=\psi_{y y}(\mathrm{t}, \mathrm{x}, \mathrm{y})=-\psi_{x}(\mathrm{t}, \mathrm{x}, \mathrm{y})$

For some scalar function, $\psi$ called the stream function. As we know vortisity 
$\Omega=\nabla \times v=-\nabla^{2} \psi 2$

Curl of the Navier Stokes equation gives

$\Omega, \mathrm{t}+\nabla(\Omega \times v)+2 \nabla \times(\omega \times v)+$

$\nabla \times(\omega(\omega \times r))=v \nabla^{2} \Omega$

Here, $\omega \times(\omega \times \mathrm{r})=(\omega . \mathrm{r}) \omega-\omega^{2} r$

and $\nabla \times(\omega \times(\omega \times \mathrm{r}))=0$

Since $\omega$ is assumed to be constant, thus, the Navier Stokes equation gives us

$(\Omega, \mathrm{t}+\nabla \mathrm{x}(\Omega \times)+2 \nabla \times(\omega \times v)$

$$
=v \nabla^{2} \Omega
$$

Using above equations, we construct a MHD Antenna with appropriate beam function. Here, we take velocity field $v(\mathrm{t}, \mathrm{r})$, Electric field $\mathrm{E}(\mathrm{t}, \mathrm{r})$

The Magnetic field B ( $t$, r). These quantities can be computed with coupled solution of Navier-Stokes equation and Maxwell's equations. With $\sigma$ denoting the conductivity of the fluid, the current density is given by

$\mathrm{J}=\sigma(\mathrm{E}+v \times \mathrm{B})$

The fields E, B, $v$ can be obtained on solving coupled

Maxwell's and Navier Stokes equations

$\nabla \times \mathrm{E}=\mu \mathrm{H}, \mathrm{t}$

$\nabla \times \mathrm{H}=\sigma(\mathrm{E}+v \times \mathrm{B})+\epsilon \mathrm{E}, \mathrm{t}$

$\Omega, \mathrm{t}+\nabla \times(\Omega \times \mathrm{v})$

$=v \nabla^{2} \Omega+\nabla^{2} v+\rho^{-1} \sigma \nabla \times((\mathrm{E}+v \times \mathrm{B}) \times \mathrm{B}$

$\Omega=\nabla \times v$

$\mathrm{B}=\mu \mathrm{H}$

$\nabla . \nu=0$

There exists a vector field $\psi$ such that

$v=\nabla \times \psi$ and $\psi$ can be chosen such that

$\nabla . \psi=0$

Let $\mathrm{V}$ denote the region of space over which the conducting fluid is presented. The Magnetic vector potential produced by the fluid can be taken as

$$
\mathrm{A}(\mathrm{t}, \mathrm{r})=\frac{\mu}{d \pi r} \int_{E} f\left(\mathrm{t}-\frac{r}{c}+\frac{\rho_{-} r^{s}}{\varepsilon}, r^{r}\right) \mathrm{d}^{\mathrm{g}} r^{r}
$$

Suppose, we look at the $\sigma(v \times \mathrm{B})$ term in J. Fourier analysis of $v$ and $\mathrm{B}$ shall give us

$v(\mathrm{t}, \mathrm{r})=\int_{R} v(\omega, \mathrm{r}) \exp (\mathrm{j} \omega \mathrm{t}) \mathrm{d} \omega$

$\mathrm{B}(\mathrm{t}, \mathrm{r})=\int_{R} B(\omega, \mathrm{r}) \exp (\mathrm{j} \omega \mathrm{t}) \mathrm{d} \omega$

$\mathrm{v} \times \mathrm{B}=$

$\int \exp f\left(\omega_{1}, \omega_{2}\right) t\left(\theta\left(\omega_{1}, \mathrm{r}\right) \times B\left(\omega_{2}, \mathrm{r}\right)\right) \mathrm{d} \omega_{1} \mathrm{~d} \omega_{2}$

Current density

$\mathrm{J}(\mathrm{t}, \mathrm{r})=\sigma(v \times \mathrm{B})$

$\mathrm{J}(\omega, \mathrm{r})=\sigma \int_{E} v\left(\omega_{1}, \mathrm{r}\right) \mathrm{x} B\left(\omega-\omega_{1}, \mathrm{r}\right) \mathrm{d} \omega_{1}$

$\hat{f}\left(\omega_{\mathrm{g}} \mathrm{r}\right)=\sigma \int_{R} \theta\left(\omega_{1}, \mathrm{r}\right) \times \vec{B}\left(\omega-\omega_{1}, \mathrm{r}\right) \mathrm{d} \omega_{1}$
Amplitude of far field magnetic vector potential at the frequency $\omega$ is given by

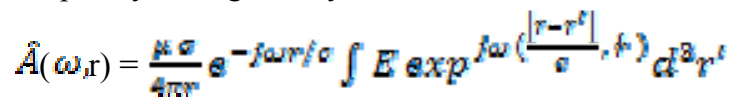

$\mathrm{A}(\omega, \mathrm{r})$

$$
=\frac{\mu}{4 \pi r} e^{-j k r} \int J(\omega, \mathrm{r}) \exp p^{\delta k\left(r-r^{k}\right)} d^{3} r^{t}
$$

Where, $\mathrm{k}=\frac{\omega}{\mathrm{c}}$

and k.r $>1$

since $\phi(\omega, \mathrm{r})$ is scalar.

$\operatorname{Div} \mathrm{A}=-\mathrm{j} \mu \epsilon \omega \phi$

$$
\Phi=\frac{j}{\mu \in a} \operatorname{Div} \mathrm{A}
$$

Energy radiated is given as

$\mathrm{E}=-\nabla \phi-\mathrm{j} \omega \mathrm{A}$

$\frac{|B|^{2} e}{4 \mu} r=$

$\int_{0}^{\pi} \int_{0}^{2 \pi}\left[\left(\frac{\alpha \omega_{0}}{4 \pi c}\right)^{2}\left|\beta \mathrm{x}_{0}\right|^{2}\left|\mathrm{~F}_{0}(\theta, \phi)\right|^{2} \frac{c}{4 \mu}+\right.$

$\left.\left(\frac{\mu \theta_{1}}{4 \pi \varepsilon}\right)^{2}\left|f \times J_{1}\right|^{2}\left|F_{1}(\theta, \phi)\right|^{2} \frac{\varepsilon}{4 \mu}\right] \sin \theta d \theta d \phi$

\section{FLUID FRAME}

$\mathrm{E}_{\mathrm{x}} \quad$ Electric field in $\mathrm{X}$ direction

$\mathrm{B}_{y} \quad$ Magnetic field in $\mathrm{Y}$ direction

$\mathrm{E}=E_{x} \hat{x}$

$\mathrm{B}=B_{y} f$ and $\mathrm{v}_{x}, \mathrm{v}_{\mathrm{y}} \mathrm{v}_{\mathrm{z}}$ (velocity)

$\frac{\mathrm{d} \mathrm{v}_{\mathrm{K}}}{\mathrm{dt}}=\frac{Q}{m}\left(E_{x}-v_{\mathrm{z}} B_{y}\right)$

$\frac{d v_{\mathrm{y}}}{d t}=0$

$\frac{d v_{z}}{d t}=\frac{Q}{m}\left(v_{x} B_{y}\right)$

$\frac{d}{d t}\left[\begin{array}{l}\gamma_{X} \\ w_{Z}\end{array}\right]=\frac{Q}{m} B_{Y}\left[\begin{array}{cc}0 & -1 \\ 1 & 0\end{array}\right]\left[\begin{array}{l}\gamma_{X} \\ w_{Z}\end{array}\right]+\frac{Q}{m} \mathbf{E}_{Y}\left[\begin{array}{l}1 \\ 0\end{array}\right]$

Let, $\frac{Q}{\mathrm{~m}}=\boldsymbol{\alpha}$

$v_{\mathrm{X}}=\frac{w_{7}}{m B_{y}}$

And

$\frac{1}{\mathrm{mB}_{\mathrm{y}}} \mathrm{v}_{\mathrm{z}}{ }^{\mathrm{II}}=\frac{\mathrm{Q}}{\mathrm{m}}\left(\mathrm{E}_{\mathrm{x}}-\mathrm{v}_{\mathrm{z}} \mathrm{B}_{\mathrm{y}}\right.$

$\mathrm{v}_{\mathrm{x}}{ }^{\mathrm{Ex}}=-\alpha \mathrm{B}_{\mathrm{y}}{ }^{\mathrm{z}}{ }^{\mathrm{T}}$

$v_{x}{ }^{R T}=-\left(\alpha B_{y}\right)^{2} y_{x}$ 


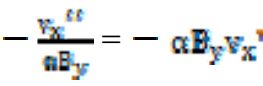

Hence, fluid frame velocity shall be

$v_{x}(t)=A \operatorname{Cos}\left(\alpha B_{y}, t+\phi\right)=A \operatorname{Cos}(\omega t+\phi)$

$\alpha B_{y}=\omega=\frac{B_{y}}{m}$

$\mathrm{v}_{\mathrm{R}}(0)=0$

$\mathrm{v}_{\mathrm{x}}{ }^{\mathrm{s}}(0)=\frac{\mathrm{g}}{\mathrm{m}} \mathbf{E}_{\mathrm{x}}$

$\mathrm{A} \operatorname{Cos} \phi=0$

Where $\phi=\frac{\pi}{2}$

$\mathrm{v}_{\mathrm{x}}(\mathrm{t})=\mathrm{A} \operatorname{Sin}(\omega \mathrm{t})$

$\mathrm{A} \omega=\frac{\mathrm{Q}}{\mathrm{E}_{\mathrm{X}}}$

$\mathrm{A}=\frac{\mathrm{Q}}{\mathrm{m}} \frac{\mathrm{E}_{x}}{\mathrm{~B}} \frac{\mathrm{m}}{\mathrm{B}_{y}}=\frac{\mathrm{E}_{x}}{\mathrm{~B}_{y}}$

$v_{x}(t)=\frac{E_{x}}{E_{y}} \operatorname{Sin}(\omega t)$

$v_{x}=\frac{d v_{x}}{d t}=$

$\frac{\mathrm{Q}}{\mathrm{m}}\left(\mathrm{E}_{\mathrm{x}}-\mathrm{w}_{\mathrm{z}} \mathrm{E}_{\mathrm{Y}}\right)=\frac{\mathrm{E}_{\mathrm{x}}}{\mathrm{E}_{\mathrm{y}}} \operatorname{Cos}(\omega \mathrm{t})$

$\mathbf{E}_{\mathrm{x}}-\mathrm{v}_{\mathrm{z}} \mathrm{E}_{\mathrm{Y}}=\mathrm{E}_{\mathrm{K}} \operatorname{Cos}(\omega \mathrm{t})$

$\mathrm{v}_{z}=\frac{\mathrm{E}_{x}}{\mathrm{~B}_{y}} \operatorname{Cos}(1-\omega \mathrm{t})$

The fluid velocity can be defined as

$v=\frac{E_{1}}{B_{u}}\left(t \operatorname{Cos}\left(\omega_{0} t\right)+\hat{z}\left(1-\operatorname{Cos}\left(\omega_{0} t\right)\right)\right.$

If $\mathbf{E}_{\mathrm{x}}=\mathrm{E}_{\mathrm{n}}$ and $\mathbf{B}_{\mathrm{y}}=\mathrm{B}_{0}$

Then, $\mathbb{E}_{0} \hat{\mathrm{R}}+\mathrm{E}_{1} \operatorname{Cos}(\omega \mathrm{t})=\mathrm{QB}_{0} / \mathrm{m}$

Current density in this case shall be

$J=\sigma\left(\mathbb{B}_{0} \hat{A}+\mathbb{H}_{1} \operatorname{Cos}(\omega, t)+\frac{E_{g}}{\theta_{\mathbb{Q}}}\left(\hat{x} \operatorname{Cos}\left(\omega_{0} t\right)+\right.\right.$

And

$$
\left.\hat{z}\left(1-\operatorname{Cos}\left(\omega_{0} t\right)\right) \times \mathbf{B}_{0} \hat{\mathrm{Y}}\right)
$$

$$
\begin{aligned}
& \left|\beta \times J_{0} \|^{2}=V_{0}\right|^{2}-\left(\beta_{0} J_{0}\right)^{2} \\
& =\left(J_{0 x}{ }^{2}+J_{0 y}{ }^{2}+J_{0 z} z^{2}\right)- \\
& \left(J_{0 x} \operatorname{Cos} \phi \operatorname{SIn} \theta+J_{0 y} \operatorname{Sin} \phi \operatorname{Sin} \theta+J_{0 z} \operatorname{Cos} \theta\right)^{2}
\end{aligned}
$$

Magnetic vector potential( A) can thus be evaluated as $A=$

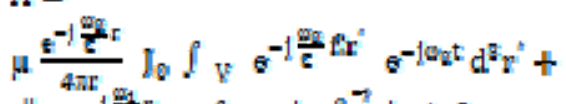

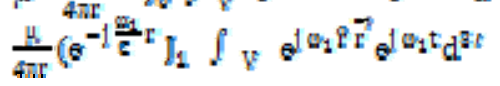

From coordinate transformation we can write

$\mathrm{x}^{r}=\rho^{r} \cos \phi^{r}$

$y^{r}=\rho^{r} \operatorname{Sin} \phi^{r}$

$\mathbf{z}^{t}=\mathbf{z}^{t}$

Electric field Intensity radiation pattern in the far field can be computed as

$$
\begin{aligned}
& \mathrm{E}=\frac{\mu}{d \pi}\left(j \frac{\omega_{\mathrm{a}}}{c}\right) \hat{r} \times l_{0} \theta^{-j \log _{0} \mathrm{~F}} \mathrm{~F}_{0}(\theta, \phi)+
\end{aligned}
$$

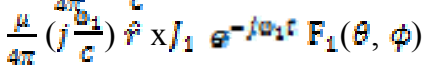

$\frac{|B|^{2} G}{4 \mu} r^{2}=$

$\left.\int_{0}^{\pi} \int_{0}^{2 \pi}\left[\left(\frac{\phi \omega \omega_{0}}{4 \pi c}\right)^{2} \mid \beta \times\right]_{0}\right|^{2}\left|F_{0}(\theta, \phi)\right|^{2} \frac{c}{4 \mu}+$

$\left.\left(\frac{\mu \omega_{1}}{4 \pi \varepsilon}\right)^{2}\left\|f \times J_{1}\right\|^{2}\left|\mathbb{F}_{1}(\theta, \phi)\right|^{2} \frac{\varepsilon}{4 \mu}\right] \sin \theta d \theta d \phi$

Where, $J_{0}=\sigma E_{0}(\hat{x}+z)$ ( current due to initial voltage )

$J_{1}=\sigma E_{1} \hat{y} \quad$ (current due to excitation of RF input )

also

$J_{0}=I_{0 x} x+J_{0 y} y+J_{D z} z$

$J_{1}=J_{L x} \hat{x}+J_{L y} y+J_{L z} z$

Radiation Intensity pattern

$F_{0}(\theta, \phi)=\int e^{-j \frac{\omega_{0}}{\varepsilon}\left(x^{\prime} \operatorname{sos} \phi \sin \theta+y \sin \phi \sin \theta+z^{2} \cos \theta\right)} d x^{5} d y^{5} d z$

$d x^{\prime} d y^{\prime} d z^{\prime}=\rho^{\prime} d \rho^{\prime} d \phi^{\prime} d z^{\prime}$

When input is applied at the center, Radiation field pattern $f(\theta, \emptyset)=$

$\int_{a}^{b} \int_{0}^{2 \pi} \int_{-\frac{1}{2}}^{+\frac{1}{2}} \exp \left(\frac{\omega_{\theta}}{\mathrm{c}}\left(\rho^{r} \operatorname{Sin} \theta \operatorname{Cog} \phi \operatorname{Cos} \phi^{r}+\rho^{r} \operatorname{Sin} \theta\right)\right.$
$\left.\left.+\mathrm{z}^{\mathrm{r}} \operatorname{Cos} \theta\right)\right) \rho^{\mathrm{r}} \mathrm{d} \rho^{r} \delta \phi^{r} \mathrm{~d} z^{r}$

Radiation Pattern can be written as

$\iint_{0 \theta}^{\pi 2 \pi}\left[\left(\frac{\phi \omega \omega_{0}}{4 \pi c}\right)^{2}\left|p_{x} J_{0}\right|^{2}\left|F_{0}(\theta, \phi)\right|^{2}\right.$

$\left.+\left(\frac{\omega \omega_{1}}{4 \pi \varepsilon}\right)^{2}\left|p_{\mathrm{K}} /_{1}\right|^{2}\left|F_{1}(\theta, \phi)\right|^{2}\right] \sin \theta d \theta d \phi$

Only real part if we consider

$R_{\theta}\left[J_{0} e^{j \omega_{0} t}+J_{1} e^{j \omega_{2} t}\right]$

Here, we get multiple harmonics in the results. These harmonics can be filtered out. For any particular frequency application, unwanted frequency can be rejected by making use of proper filtering techniques.

INPUT IMPEDANCE OF THE ANTENNA

$Z_{\text {in }}=\int_{V}\left(E \cdot J /|\mathrm{I}|^{\wedge} 2\right) \mathrm{dV}$

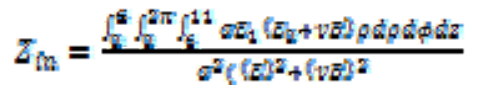

Where $\mathrm{J}$ is current density, E is electric field applied, I is load current, $\mathrm{v}$ velocity of the fluid and $\mathrm{V}$ represents volume integral. 


\section{RESULTS AND DISCUSSION}

Two cylindrical tubes of Propylene Random Copolymer Pipes (PPR) of diameters of $10.5 \mathrm{~cm}$ and $6.2 \mathrm{~cm}$ of height $6.5 \mathrm{~cm}$ respectively mounted on copper ground plane of $35 \mathrm{~cm}$ diameter as shown in figure 1. Standard SMA is mounted at the center of y axis for RF excitation. Two tin electrodes for biasing plasma are mounted in such a way that filed is orthogonal to RF input and magnetic field making direct contact with the conducting fluid. Bias voltage to the electrodes of tin $1.2 \mathrm{~cm} \times 4.1 \mathrm{~cm}$ mounted on the inner wall of the tube, making physical contact with the conducting fluid, is given through variable DC source in the range $5-25$ volts with BIAS TEE arrangement safety of VNA . SMA connector male part (protruded) acts as probe inserted into plasma. The probe dimensions are $0.08 \mathrm{~cm}$ in diameter and $0.75 \mathrm{~cm}$ long. This probe is inserted in such a way that it makes direct contact with the conducting fluid in dielectric resonator. Two permanent bar magnets having dimensions $15 \mathrm{~cm} \mathrm{x} 4 \mathrm{~cm} \times 2 \mathrm{~cm}$ are placed perpendicular to the electric field, so as to produce Lorentz force, resulting into fluid flow. Here, DRA (Dielectric Resonator Antenna) is filled with saline water having TDS (total dissolved salt) between 4000 to 12000 . Adding common salt to water will provide variable TDS. Volume of saline water decides geometrical dimensions of DRA to produce desired resonant frequency. The resonator column effects resonant frequency. Radiating resistance and resonant frequency depends on largely on shape and geometrical dimensions of fluid inside the tube and nano particles of the fluid.

$40 \mathrm{GHz}$ Network Analyser VNA-L5230 have been used to measure return loss and resonant frequency. The Radiation patterns and Gain measurements were carried out at near field test facility of Bharat Electronics an Ministry of Defence, for resonant frequency $4.59 \mathrm{GHz}$ as shown in fig.2-5 to get $\mathrm{E}, \mathrm{H}$ and Cross fields patterns.

Measured results of prototype antenna are

Return loss $=-27.1 \mathrm{~dB}$, Gain $=9.2 \mathrm{dBi}$ and

Resonant frequency $=4.59 \mathrm{GHz}$.

We have formulated various equations based on fluid frame of our prototype design. Here, we first describe beam formation, radiating patterns and resonance frequency.

Here, we see radiation parameters of our antenna depends not only on electromagnetic fields, but also on fluid velocity field as shown in equations [1-32]. From analytical results it is observed that it generates harmonics of resonant frequency. Equations [1-32] presents mathematical concept. Figure [2-8] and table [1-2] presents measured results based on proto type and HFSS modelling. Matlab simulated results are placed in fig 6 for radiation pattern. Comparison between prototype result and numerical results has been made. Here it observed that varying fluid volume can result into tuneable resonant frequency. The adaptable permeability and permeability is described in section three. This can result into possible tuning of polarisation. As results have shown generation of harmonics with fundamental frequency, hence with proper filtering, this antenna can made to operate at any desired frequency from the harmonics. The effects of magnetic bias on antenna have been investigated. The principle of this class of antenna is dielectric resonator, where salt (in solution) and electric field modifies the dielectric properties. We have varied fluid salinity, magnitude electric field and magnetic field, fluid height for all possible combinations as shown in tables 1-2. Here, we see chemical properties of fluid, shape of tube, effective biasing voltage and magnetic field conditions changes antenna parameters which reflected in tables 1-2.

Here ionized currents contribute to radiate energy in conducting fluid. The tube was applied external magnetic field which interacts with electric field to produce Lorentz forces, resulting in fluid flow with velocity v. Now there are three main fields i.e. electric field, magnetic field and velocity fields, which are responsible for the possible radiations. The radiated energy and its pattern are function of RF input excitation, fields applied, fluid shape and nano particle of fluid.

Hence an adaptive mechanism can be built in antenna to produce versatility in radiation pattern, due to dynamic fluid perturbations. We shall consider thefluid in coordinates rotating with it and mechanical equations of motion must include effects of centrifugal and Coriolis forces. Wave are also due to Coriolis forces $(2 \mathrm{v} \times \Omega)$, which occur in rotation.

Here we first describe beam formation, radiating patterns and resonance frequency. Radiation patterns in the far fields depend not only on electromagnetic field but also on fluid velocity. Gain measurements with and without electric and magnetic fields were carried out. It got increased from $6.22 \mathrm{dBi}$ to 9.10 $\mathrm{dBi}$ on applying electric fields at a value of $5.2 \mathrm{~V}, \mathrm{DC}$ to $7.5 \mathrm{~V}$. Also considerable change in Return loss were seen i.e. S11 measured -18.7 dB without Electric and Magnetic fields and increased to $-21.7 \mathrm{~dB}$ DC bias. Return loss further improved to $-27.7 \mathrm{~dB}$ when both electric and magnetic fields were applied $40 \mathrm{~dB}$ isolation was seen in cross polarization results. The above quoted results are not optimized. No corrosion takes place in this type of antenna. Obtained results indicated, reconfigurability, frequency agility and tuning of Gain possibility. 


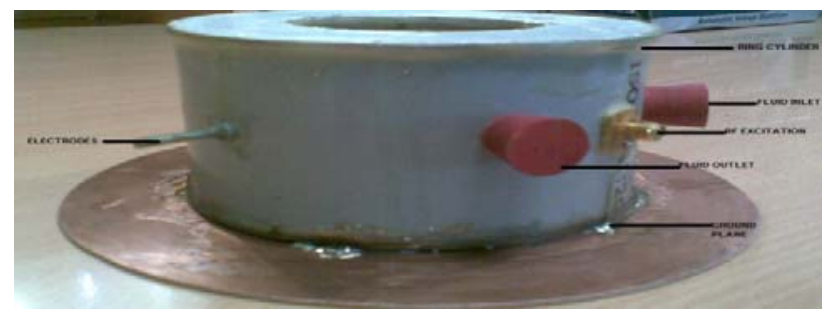

Figure 1.

MHD Antenna Proto- Type Model

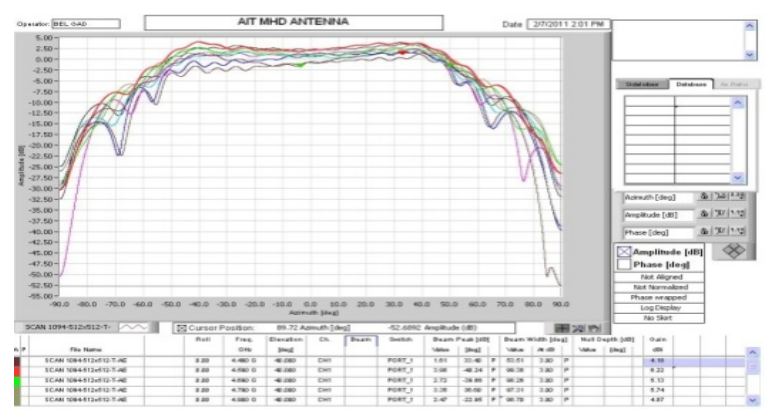

Figure 2.

Azimuth Pattern

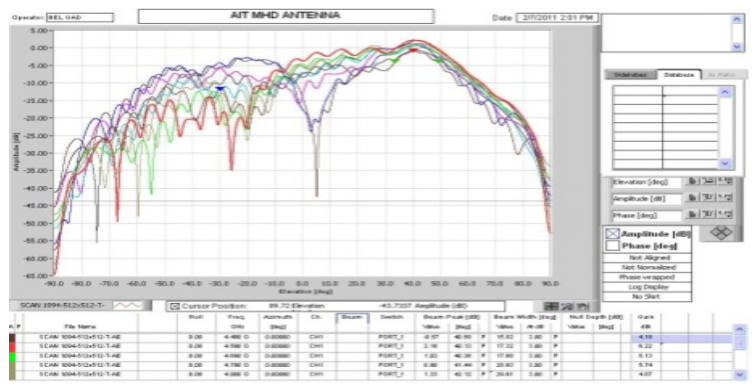

Figure 3. Elevation Pattern

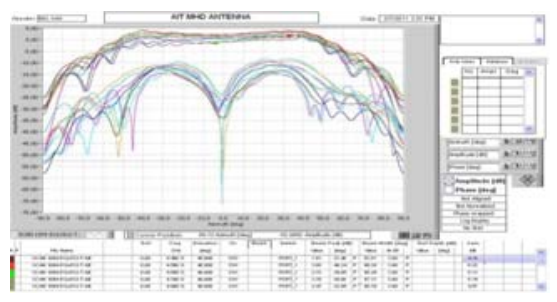

Figure 4. Cross Polarization pattern

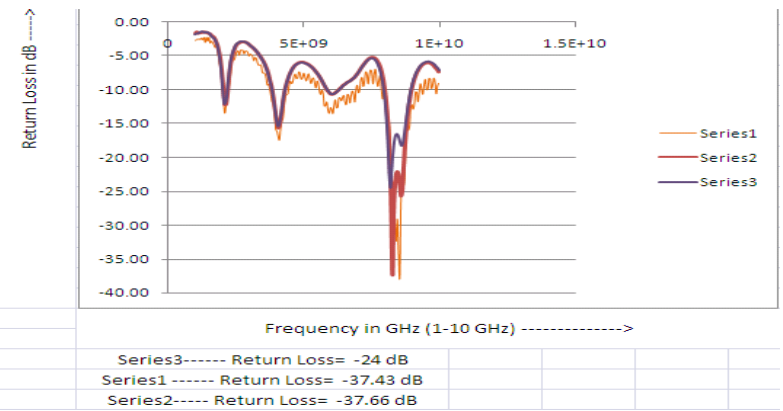

Figure 5. Return loss -24.dB with only electric field. 37.66 when both electric and magnetic field applied.

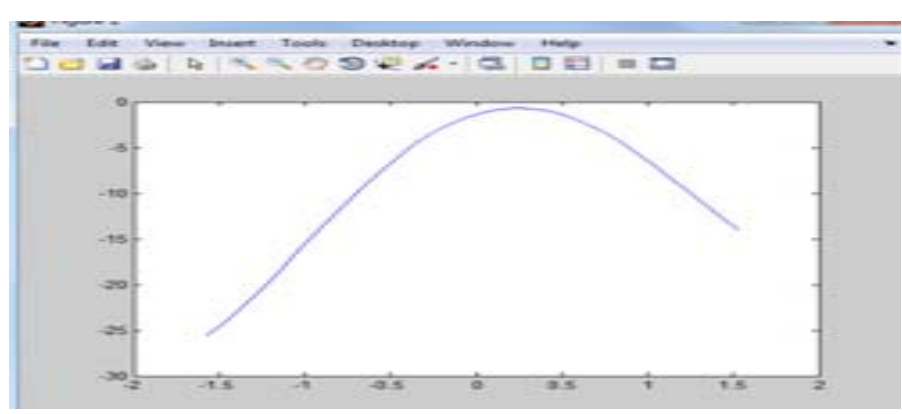

Figure 6. Matlab Plot for E field Radiation Pattern

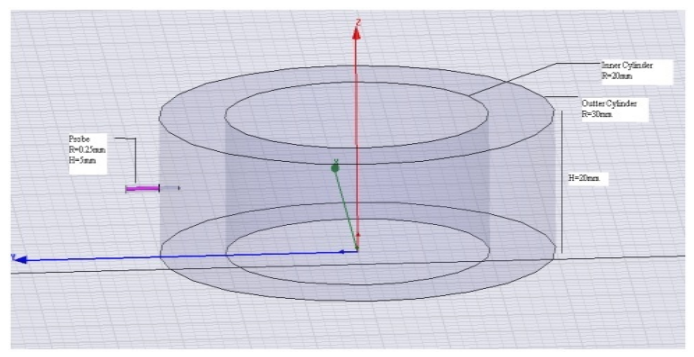

Figure 7. HFSS Model of cylindrical antenna
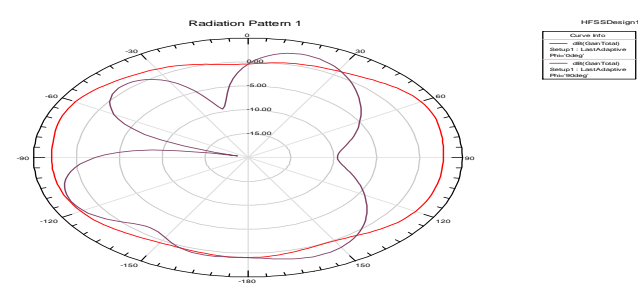

Figure 8. HFSS Radiation Pattern 
Table 1. Resonant Freq. and Gain relation with $\mathrm{r} / \mathrm{h}$ ratio

\begin{tabular}{|l|l|l|l|l|l|}
\hline $\begin{array}{l}\text { Radius } \\
(\mathrm{mm})\end{array}$ & $\begin{array}{l}\text { Height } \\
(\mathrm{mm})\end{array}$ & \multicolumn{2}{|l|}{ Sea water( $\mathrm{\epsilon}=81)$} & \multicolumn{2}{l|}{ Mercury $(\epsilon=1.00074)$} \\
\hline & & $\begin{array}{l}\text { Theoreti } \\
\text { cal } \\
(\mathrm{GHz})\end{array}$ & $\begin{array}{l}\text { Simulated } \\
(\mathrm{GHz})\end{array}$ & $\begin{array}{l}\text { Theoretical } \\
(\mathrm{GHz})\end{array}$ & $\begin{array}{l}\text { Simulated } \\
(\mathrm{GHz})\end{array}$ \\
\hline 25 & 20 & 9.7 & 10.84 & 10.76 & 11.16 \\
\hline 25 & 30 & 7.52 & 8.85 & 8.74 & 7.24 \\
\hline 25 & 40 & 7.07 & 8.75 & 7.97 & 7.14 \\
\hline 30 & 20 & 10.24 & 11.29 & 10.5 & 11.02 \\
\hline 30 & 30 & 5.97 & 4.88 & 6.83 & 5.2 \\
\hline 30 & 40 & 4.9 & 4.66 & 5.53 & 4.54 \\
\hline
\end{tabular}

Table 2. Sea water and mercury results

\begin{tabular}{|l|l|l|l|}
\hline $\begin{array}{l}\text { (r) } \\
\text { Radius of } \\
\text { Antenna(mm) }\end{array}$ & $\begin{array}{l}\text { (h) } \\
\text { Height(mm) }\end{array}$ & $\begin{array}{l}(\mathrm{f}) \\
\text { Resonant } \\
\text { frequency } \\
(\mathrm{GHz})\end{array}$ & \begin{tabular}{l} 
Gain(dBi) \\
\hline 25
\end{tabular} \\
\hline 25 & 30 & 8.9 & 5 \\
\hline 25 & 40 & 8.75 & 7 \\
\hline 27 & 50 & 8.65 & 8 \\
\hline 27 & 30 & 25.5 & 10 \\
\hline & 40 & 21.2 & 11 \\
\hline
\end{tabular}

\section{CONCLUSION}

Due to stealth property fluid being plasma, it conducts only in excited state, hence most suitable for Military applications. This antenna has advantage of corrosion resistant, frequency agility and better coupling of electromagnetic signal. It can also be used for biomedical applications like a fluid bracelet to monitor health parameters.

As a future work, with integration of microcontroller based MHD fluid pump, height and shape of the fluid, can be regulated. This will provide reconfigurability agility. More work towards micro-fluidic frequency reconfiguration, fluidic tuning of matching networks for bandwidth enhancement need to be explored. We have solved this problem for saline water and mercury. It can be tried out for other plasma materials like ionized gases and Gel based fluids.

\section{REFERENCES}

[1] Rajveer S Yaduvanshi and Harish Parthasarathy, "Design, Development and Simulations of MHD Equations with its proto type implementations"(IJACSA) International Journal of Advanced Computer Science and Applications,Vol. 1, No. 4, October 2010.

[2] Rajveer S Yaduvanshi and Harish Parthasarathy, "EM Wave transport 2D and 3D investigations" (IJACSA) International Journal of Advanced Computer Science and Applications,Vol. 1, No. 6, December 2010.

[3] Rajveer S Yaduvanshi and Harish Parthasarathy, "Exact solution of 3D Magnetohydrodynamic system with nonlinearity analysis" Jan 2011, IJATIT.

[4] EM Lifshitz and LD Landau, "Theory of Elasticity, 3rd edition Elsevier.

[5] EM Lifshitz and LD Landau,“ Classical theory of fields , 4th edition Elsevier.

[6] Bahadir, A.R. and T. Abbasov (2005), "A numerical investigation of the liquid flow velocity over an infinity plate which is taking place in a magnetic field" International journal of applied electromagnetic and mechanics 21, 1-10.

[7] EM Lifshitz and LD Landau, "Electrodynamics of continuous media" Butterworth-Heinemann.

[8] EM Lifshitz and LD Landau," Fluid Mechanics" Vol. 6 Butterworth-Heinemann.

[9] EM Lifshitz and LD Landau," Theory of Fields" Vol. 2 Butterworth-Heinemann.

[10] JD Jackson, "Classical Electrodynamics" third volume, Wiley.

[11] CA Balanis," Antenna Theory, Wiley

[12] Gregory H. Huff, Member, IEEE, David L. Rolando, Student Member, IEEE, Phillip Walters ,Student Member, IEEE and Jacob McDonald, "A Frequency Reconfigurable Dielectric Resonator Antenna using Colloidal Dispersions "IEEE ANTENNASAND WIRELESS PROPAGATION LETTERS, VOL. 9, 2010.

[13] Fayad H and Record P, Wideband salt water Antenna IEEE conf on wide band and multi band antenna and arrays, Sep 2005

[14] Buncher R ,Hepter GT and May MP , Dielectric relaxation of acquous $\mathrm{NaCl}$ solutions J Phy, Chem a 1999103 PP1-9. 